\title{
Caffeine Affects Myotube Size As Well As Regulates Protein Degradation and Protein Synthesis Pathways in C2C12 Skeletal Muscle Cells
}

\section{$\operatorname{AUTHOR}(\mathrm{S}):$}

Egawa, Tatsuro; Ohno, Yoshitaka; Goto, Ayumi; Sugiura, Takao; Ohira, Yoshinobu; Yoshioka, Toshitada; Hayashi, Tatsuya; Goto, Katsumasa

\section{CITATION:}

Egawa, Tatsuro ...[et al]. Caffeine Affects Myotube Size As Well As Regulates Protein Degradation and Protein Synthesis Pathways in C2C12 Skeletal Muscle Cells. Journal of Caffeine Research 2016, 6(2): 88-96

\section{ISSUE DATE:}

2016-05-24

URL:

http://hdl.handle.net/2433/225060

\section{RIGHT:}

Final publication is available from Mary Ann Liebert, Inc., publishers

http://doi.org/10.1089/jcr.2015.0034.; This is not the published version. Please cite only the published version.; この論文は出版社版でありません。引用の際には出版社版をご確 認ご利用ください。 
Caffeine affects myotube size as well as regulates protein degradation and protein synthesis pathways in C2C12 skeletal muscle cells

Tatsuro Egawa ${ }^{1,2}$, Yoshitaka Ohno ${ }^{3}$, Ayumi Goto ${ }^{2}$, Takao Sugiura ${ }^{4}$, Yoshinobu Ohira ${ }^{5}$, Toshitada Yoshioka ${ }^{6}$, Tatsuya Hayashi ${ }^{2}$, Katsumasa Goto ${ }^{1^{*}}$

1 Department of Physiology, Graduate School of Health Sciences, Toyohashi SOZO University, Toyohashi, Aichi, Japan

2 Laboratory of Sports and Exercise Medicine, Graduate School of Human and Environmental Studies, Kyoto University, Kyoto, Japan

3 Laboratory of Physiology, School of Health Sciences, Toyohashi SOZO University, Toyohashi, Aichi, Japan

4 Department of Exercise and Sports Physiology, Faculty of Education, Yamaguchi University, Yamaguchi, Japan

5 Graduate School of Health and Sports Science, Doshisha University, Kyotanabe, Kyoto, Japan

6 Hirosaki Gakuin University, Hirosaki, Aomori, Japan

Running head: Regulation of skeletal muscle size by caffeine

*Corresponding author: Katsumasa Goto, Ph.D.

Laboratory of Physiology, Graduate School of Health Sciences, Toyohashi SOZO University, 20-1 Matsushita, Ushikawa-cho, Toyohashi, Aichi, 440-8511, Japan 
TEL: +81-50-2017-2272, FAX: +81-532-55-0803

Email: gotok@sepia.ocn.ne.jp 


\section{ABSTRACT}

Background: Caffeine has been implicated in energy metabolism regulation in skeletal muscle. However, it is unclear whether caffeine affects the regulation of skeletal muscle size. In the present study, we evaluated the effect of caffeine on muscle size as well as accompanied changes of ubiquitin-proteasome system and Akt/mammalian target of rapamycin/p70 s6 kinase (p70S6K) signaling.

Methods: Differentiated C2C12 myotubes were incubated with caffeine $(0,0.1,1.0,3.0$ $\mathrm{mM}$ ) for $24 \mathrm{~h}$. We then estimated the protein content, myotube diameter, and the expression levels of muscle RING finger 1 (MuRF1) mRNA, atrogin-1/muscle atrophy F-box (MAFbx) mRNA, K48-linked polyubiquitin, phosphorylated 5'AMP-activated protein kinase (AMPK) $\alpha$ Thr $^{172}$, 72-kDa heat shock protein (HSP72), HSP72 mRNA, inhibitor $\kappa \mathrm{B} \alpha(\mathrm{I} \kappa \mathrm{B} \alpha)$, phosphorylated forkhead box class O3a (FoxO3a) $\mathrm{Ser}^{253}$, myogenin mRNA, microRNA(miR)-23a, phosphorylated Akt Ser ${ }^{473}$, and phosphorylated p70 s6 kinase (p70S6K) Thr ${ }^{389}$.

Results: Protein content and myotube diameter were lower in myotubes treated with caffeine ( $\geq 1 \mathrm{mM})$ compared with untreated cells. The expression levels of MuRF1 and atrogin-1/MAFbx mRNA and K48-linked polyubiquitin were increased by caffeine

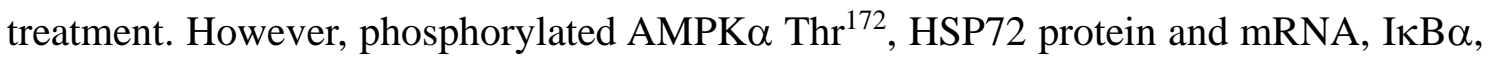
phosphorylated FoxO3a Ser ${ }^{253}$, and miR-23a expression were not affected by caffeine treatment. Myogenin mRNA expression was upregulated in response to caffeine treatment. The expressions of phosphorylated Akt $\operatorname{Ser}^{473}$ and p70S6K Thr ${ }^{389}$ were suppressed by caffeine.

Conclusions: Caffeine might affect muscle size by stimulating ubiquitin-proteasome system and inhibiting Akt/mTOR/p70S6K signaling, partly via an AMPK-independent 
mechanism.

Keywords: 5'AMP-activated protein kinase, heat shock protein, muscle RING finger 1, myogenin, skeletal muscle plasticity 


\section{INTRODUCTION}

Caffeine is a xanthine alkaloid, which has been implicated in energy metabolism regulation in skeletal muscle. Particularly, 5'AMP-activated protein kinase (AMPK) is considered to be a crucial mediator of caffeine-mediated metabolic changes in skeletal muscle. Several studies have suggested that caffeine ( $\geq 1 \mathrm{mM})$ acutely stimulates AMPK in rat skeletal muscle $e^{1-8}$ and that caffeine-stimulated glucose transport is reduced in AMPK kinase-dead mice ${ }^{1}$. Ding et al. ${ }^{9}$ found that caffeine (1 and $5 \mathrm{mM}$ ) treatment for 4 days (5 h/day) increased mitochondrial biogenesis via an AMPK-dependent mechanism in skeletal muscle cells. Furthermore, Mathew et al. ${ }^{10}$ demonstrated that caffeine (10 mM) treatment for $6 \mathrm{~h}$ stimulated autophagy in skeletal muscle cells, which was attenuated in the presence of an AMPK inhibitor.

Recently, AMPK has been considered to be a key factor that modulates skeletal muscle mass. It was reported that elevated AMPK activity was involved in a diminished capacity for hypertrophy in aged rat skeletal muscle $\mathrm{e}^{11,12}$ and that overload-induced muscle hypertrophy was accelerated in AMPK $\alpha 1$-deficient mice compared with the wild-type mice $^{13}$. Akt/mammalian target of rapamycin (mTOR)/p70 S6 kinase (p70S6K), a central signaling modulator of protein synthesis ${ }^{14}$, is considered to be a potent signaling pathway for AMPK-mediated regulation of muscle hypertrophy. On the other hand, in our previous study $^{15}$, we demonstrated that AMPK-associated suppression of muscle hypertrophy in skeletal muscle cells was through rather activating protein catabolic pathway than inhibiting protein synthesis pathway.

The ubiquitin-proteasome system is a crucial regulatory mechanism for protein catabolism in skeletal muscle. The key enzymes in this pathway are E3 ubiquitin ligases, which are responsible for protein ubiquitination. Two muscle-specific ubiquitin ligases, 
i.e., muscle RING finger 1 (MuRF1) and atrogin-1/muscle atrophy F-box (MAFbx), are considered to be involved in skeletal muscle atrophy ${ }^{16,17}$. Previous studies have demonstrated that AMPK activation stimulates protein degradation, which is accompanied by the upregulation of MuRF1 and atrogin-1/MAFbx mRNA expression in skeletal muscle cells ${ }^{18,19}$. In accordance with these researches, we ${ }^{15}$ demonstrated that pharmacological AMPK activation altered muscle size through ubiquitin-proteasome system and that these responses were not induced in AMPK-knockdown cells in vitro. Furthermore, our recent study in vivo ${ }^{20}$ has shown that the ubiquitin-proteasome system was not activated during unloading-induced muscle atrophy in muscle, where the AMPK activity decreased. Overall, AMPK-induced activation of ubiquitin-proteasome system appears to be involved in the regulation of muscle size.

Thus, the results of previous studies strongly suggest that caffeine also activates the ubiquitin-proteasome system and that it affects the skeletal muscle size through AMPK activation. Therefore, in the present study, we aimed to evaluate the effect of caffeine on muscle size and accompanied changes of the ubiquitin-proteasome system in skeletal muscle cells. In addition, we have previously shown that caffeine inhibited Akt/mTOR/p70S6K signaling in rat skeletal muscle ${ }^{7,21}$, indicating that caffeine may inhibit protein synthesis pathway. Therefore, to confirm whether caffeine affects protein synthesis pathway, we also evaluate the effect of caffeine on Akt/mTOR/p70S6K signaling. 


\section{MATERIALS AND METHODS}

Cell culture and treatment.

Cell culture was performed as described previously ${ }^{15}$. Briefly, mouse myoblast C2C12 cells were cultured on 6-well culture plates with type I collagen-coated surface (Biocoat, Becton-Dickinson Labware, Franklin Lakes, NJ). Cells were maintained in growth medium comprising Dulbecco’s modified Eagle’s medium (DMEM, Invitrogen, Rockville, MD) supplemented with 10\% heat-inactivated fetal bovine serum containing high glucose (4.5 g/l glucose, $4.0 \mathrm{mM} \mathrm{L}$-glutamine, without sodium pyruvate) to allow proliferation under a humidified atmosphere with $95 \%$ air and $5 \% \mathrm{CO}_{2}$. After reaching confluence, the culture medium was replaced with differentiation medium comprising DMEM supplemented with 2\% heat-inactivated horse serum containing low glucose $(1.0$ g/l glucose, $4.0 \mathrm{mM}$ L-glutamine, and $110 \mathrm{mg} / \mathrm{l}$ sodium pyruvate) to initiate differentiation. The medium was replaced with freshly prepared differentiation medium at 2-day intervals over the course of differentiation for 5 days. Five days after the initiation of differentiation, myotubes were incubated in differentiation medium containing caffeine (Wako, Tokyo, Japan), 5-aminoimidazole-4-carboxamide-1- $\beta$-D-ribonucleoside (AICAR, Sigma, St. Louis, MO), or metformin (Sigma). To investigate the dose-dependent effects of caffeine, the myotubes were incubated in differentiation medium with or without caffeine $(0,0.1,1.0,3.0 \mathrm{mM})$ for $24 \mathrm{~h}$. Subsequently, the cells were harvested for western blot or real-time RT-PCR analyses.

Myotube diameter measurement.

Images of myotubes were visualized at $40 \times$ magnification using an inverted light microscope and captured with a camera (Olympus, Tokyo, Japan). The diameter of 
myotubes was measured using a modified version of the method described by Williamson et al. ${ }^{22}$. Four fields were chosen randomly and 150 myotubes were measured using ImageJ. The average diameter per myotube was calculated as the mean of three short-axis measurements taken along the length of the myotube.

\section{Sample preparation.}

Samples were prepared as described previously with some modifications ${ }^{23}$. Briefly, the cells in each well were rinsed twice with ice-cold phosphate-buffered saline. Next, the cells were scraped from each well into CelLytic ${ }^{\mathrm{TM}} \mathrm{M}$ cell lysis reagent (Sigma) with 1\% (v/v) protease inhibitor cocktail (Sigma) and 1\% (v/v) phosphatase inhibitor cocktail (Calbiochem, San Diego, CA). The cell lysate was sonicated and centrifuged for $15 \mathrm{~min}$ at $15,000 \times g$ and $4^{\circ} \mathrm{C}$. The supernatant was collected to determine the protein content using the Bradford technique (protein assay kit; Bio-Rad, Hercules, CA). The protein contents of the supernatant were expressed as $\mathrm{mg} / \mathrm{ml}$.

\section{Western blot analyses.}

Western blot analyses were performed as described previously ${ }^{7,23}$. Extracted samples in cell lysis reagents were solubilized in Laemmli's sample buffer and boiled for 5 min. The samples (10 $\mu$ g of protein) were separated by SDS-PAGE using 10\% polyacrylamide gel at a constant current of $35 \mathrm{~mA}$ /gel for 90 min. Bio-Rad Precision Markers (Bio-Rad Laboratories, Hercules, CA) were applied to both sides of the gel as internal controls for the transfer process or electrophoresis.

After SDS-PAGE, the proteins were transferred to polyvinylidene fluoride membranes (Hybond-P, GE Healthcare, Buckinghamshire, UK) using trans-blot cell (Bio- 
Rad) at a constant voltage of $100 \mathrm{~V}$ for $1 \mathrm{~h}$ at $4^{\circ} \mathrm{C}$. Next, the membranes were blocked for 1 h using Blocking One-P (Nacalai Tesque, Kyoto, Japan) or ECL blocking reagent (RPN418, GE Healthcare). The membranes were then incubated overnight at $4^{\circ} \mathrm{C}$ with primary antibodies: Akt $\operatorname{Ser}^{473}$ (9271, Cell Signaling Technology, Danvers, MA), Akt (9272, Cell Signaling Technology), AMPK $\alpha$ Thr $^{172}$ (2531, Cell Signaling Technology), AMPKa (2532, Cell Signaling Technology), forkhead-box class O (FoxO) 3a Ser²53 (9466, Cell Signaling Technology), FoxO3a (2497, Cell Signaling Technology), 72-kDa heat shock protein (HSP72, ADI-SPA-812, Enzo Life Sciences, Farmingdale, NY), inhibitor of $\kappa \mathrm{B} \alpha$ (I $\kappa \mathrm{B} \alpha$, 9242, Cell Signaling Technology), K48-linkage specific polyubiquitin (4298, Cell Signaling Technology), p70S6K Thr ${ }^{389}$ (9206, Cell Signaling Technology), p70S6K (9202, Cell Signaling Technology), and $\beta$-actin (4967, Cell Signaling Technology). The membranes were washed with Tris-buffered saline containing 0.1\% Tween 20 (TBS-T, $\mathrm{pH}$ 7.5) and reacted with anti-rabbit IgG (Cell Signaling Technology) for $1 \mathrm{~h}$ at room temperature. After a final wash with TBS-T, the protein bands were visualized using chemiluminescence (Wako). The signal density was measured using a Light-Capture system (AE-6971, ATTO Corporation, Tokyo, Japan).

\section{Real-time RT-PCR analyses.}

Real-time RT-PCR analyses were performed as described previously ${ }^{24}$. Briefly, total RNA was extracted from muscles using a miRNeasy Mini kit (Qiagen, Hiden, Germany) according to the manufacturer's protocol. To detect MuRF1 (Trim63) mRNA, atrogin1/MAFbx (Fbxo32) mRNA, and HSP72 (Hspa1a) mRNA, the RNA was reversetranscribed to cDNA using PrimeScript RT Master Mix (Takara Bio), and the synthesized cDNA was then subjected to real-time RT-PCR (Thermal Cycler Dice Real Time System 
IIMRQ, Takara Bio) using Takara SYBR Premix Ex Taq II (Takara Bio). To detect microRNA (miR)-23a, the RNA was reverse-transcribed to cDNA using a Mir-X ${ }^{\mathrm{TM}}$ miRNA First Strand Synthesis Kit (Clontech Laboratories, Mountain View, CA), and the synthesized cDNA was then subjected to real-time RT-PCR using a Mir-X ${ }^{\mathrm{TM}}$ miRNA qRT-PCR SYBR Kit (Clontech Laboratories). Relative fold changes in the expression levels were calculated by the comparative CT method using Takara Thermal Cycler Dice Real Time System Software Ver. 4.00. To normalize the total amount of RNA present in each reaction, 18S ribosomal RNA was used as an internal standard for MuRF1 and HSP72, and U6 for miR-23a.

The following primers were used: Trim63 (MuRF1), 5'AGGACTCCTGCAGAGTGACCAA-3' (forward) and 5'TTCTCGTCCAGGATGGCGTA-3' (reverse); atrogin-1/MAFbx (Fbxo32), 5'TGTCCTTGAATTCAGCAAGCAAAC-3' (forward) and TGTGGCCATCCATTATTTCCAG-3' (reverse); Hspa1a (HSP72), 5'CAAGAACGCGCTCGAATCCTA-3' (forward) 5' TCCTGGCACTTGTCCAGCAC-3' (reverse); $18 \mathrm{~S}$ ribosomal RNA, 5'ACTCCAAAGGGCACAACTCA-3' (forward) CCTTTAGGGACCGTTACACTA-3' (reverse); and miR-23a, 5'ATCACATTGCCAGGGATTTCC-3' (forward). The U6 primer and reverse primers for miRNA were provided with the kit.

Statistical analyses.

All values were expressed as means \pm SEM. Differences between two groups were compared using the Student's t-test. Multiple means were analyzed using Dunnett's 
multiple comparisons test. Differences in the myotube diameter distributions were analyzed using the Chi-squared test. Differences between groups were considered statistically significant at $P<0.05$. All of the statistical analyses were performed using Ekuseru-Toukei 2012 software (Social Survey Research Information, Tokyo, Japan). 


\section{RESULTS}

Caffeine inhibited myotube hypertrophy.

To determine whether caffeine affects myotube size, we measured the protein content and myotube diameter in caffeine-treated cells. The dose-response analysis demonstrated that the protein content was decreased by caffeine treatment at 1.0 and $3.0 \mathrm{mM}$ compared with no treatment (Figure 1A). Correspondingly, the myotube diameter was smaller in myotubes treated with caffeine $(1 \mathrm{mM})$ compared with no treatment (Figure 1B). Greater numbers of smaller and lower numbers of larger myotubes were observed following caffeine treatment (Chi-square test: $P<0.05$, Figure 1C).

Caffeine activated the ubiquitin-proteasome system.

To identify whether caffeine activates the ubiquitin-proteasome system, we analyzed the expression levels of MuRF1 and atrogin-1/MAFbx mRNA and K48-linked polyubiquitin in myotubes treated with or without caffeine $(1 \mathrm{mM})$. The expression levels of MuRF1 mRNA (Figure 2A), atrogin-1/MAFbx mRNA (Figure 2B) and K48-linked polyubiquitin (Figure 2C) were increased by caffeine treatment.

Caffeine did not affect AMPK activity or HSP72 expression.

In a previous study ${ }^{15}$, we showed that the AMPK-induced suppression of hypertrophy is partly mediated by the downregulation of HSP72 protein. Therefore, we investigated whether caffeine affects the phosphorylation of AMPK $\alpha \mathrm{Thr}^{172}$, which is the primary site responsible for AMPK activation ${ }^{25}$, and the expression levels of HSP72 protein and mRNA. The expression level of phosphorylated AMPK $\alpha \mathrm{Thr}^{172}$ increased (Figure 3A) whereas the expression levels of HSP72 protein (Figure 3B) and mRNA (Figure 3C) 
decreased after treatment with the pharmacological AMPK activators AICAR (0.5 mM) and metformin (2 mM). However, there were no changes in AMPK $\alpha \mathrm{Thr}^{172}$ phosphorylation and the expression levels of HSP72 protein and mRNA under caffeine treatment (Figure 3).

Caffeine increased myogenin mRNA expression but not the expression levels of I $\mathrm{B} \alpha$, phosphorylated FoxO3a Ser 253 , and miR-23a.

Next, we examined the effects of caffeine on the expression levels of molecules that directly or indirectly target MuRF1 and atrogin-1/MAFbx transcriptions. The expression levels of I $\mathrm{B} \alpha$ (Figure 4A), phosphorylated FoxO3a $\mathrm{Ser}^{253}$, a key regulatory site for the transcription activity ${ }^{26}$ (Figure 4B), and miR-23a (Figure 4C) were not altered by caffeine treatment. By contrast, myogenin mRNA expression was upregulated by caffeine (Figure 4D).

Caffeine inhibited the protein synthesis pathway.

To confirm whether caffeine affects the protein synthesis pathway, we evaluated the expression levels of phosphorylated Akt Ser ${ }^{473}$ and p70S6K Thr ${ }^{389}$, which are the primary site responsible for activations of $\mathrm{Akt}^{27}$ and $\mathrm{p} 70 \mathrm{~S} 6 \mathrm{~K}^{28}$, respectively, in myotubes treated with or without caffeine $(1 \mathrm{mM})$. Treatment with caffeine decreased phosphorylation of both Akt Ser ${ }^{473}$ (Figure 5A) and p70S6K Thr ${ }^{389}$ (Figure 5B). 


\section{DISCUSSION}

In the present study, we obtained the following novel findings related to the effects of caffeine on the regulation of skeletal muscle size. First, caffeine ( $\geq 1 \mathrm{mM})$-treated myotubes had a lower protein content and smaller myotube diameter compared with untreated myotubes (Figure 1). Second, caffeine treatment increased the expression levels of MuRF1 and atrogin-1/MAFbx mRNA and K48-linked polyubiquitin (Figure 2). Third, treatment with AICAR and metformin increased the phosphorylation of AMPK $\alpha$ Thr ${ }^{172}$ but decreased the expression levels of HSP72 protein and mRNA. Conversely, caffeine treatment had no effects on the phosphorylation of AMPK $\alpha \mathrm{Thr}^{172}$ and the expression levels of HSP72 protein and mRNA (Figure 3). Fourth, caffeine treatment increased myogenin mRNA expression but not the phosphorylation of FoxO3a $\mathrm{Ser}^{253}$, IкB $\alpha$, and miR-23a (Figure 4).

A recent study demonstrated that caffeine promotes autophagy in skeletal muscle cells $^{10}$. Autophagy is a crucial cell proteolytic system, which controls protein turnover in skeletal muscle ${ }^{29}$. In the present study, we found that caffeine increased the expression levels of MuRF1 and atrogin-1/MAFbx mRNA and K48-linked polyubiquitin (Figure 2), suggesting that caffeine also activates another proteolysis pathway, the ubiquitinproteasome system. K48-linked polyubiquitination is the best-studied type of polyubiquitination, which typically targets proteins for proteasomal degradation ${ }^{30}$, and it is synthesized by MuRF1 ${ }^{31}$ and/or atrogin-1/MAFbx ${ }^{32}$. Several signaling molecules are involved in the regulation of MuRF1 and atogin-1/MAFbx transcriptions.

AMPK stimulates myofibrillar protein degradation via the upregulation of MuRF1 and atrogin-1/MAFbx ${ }^{18,19}$. We hypothesized that caffeine may increase MuRF1 and atrogin-1/MAFbx expressions via an AMPK-dependent mechanism since caffeine is 
known to be a potent activator of AMPK. However, caffeine did not affect the phosphorylation of AMPK $\alpha \operatorname{Thr}^{172}$ (Figure 3A). A previous study found that caffeine acutely (approximately $4 \mathrm{~h}$ ) increased the phosphorylation of AMPK $\alpha \mathrm{Thr}^{172}$, but it returned to the control level by $12 \mathrm{~h}$ in skeletal muscle cells ${ }^{33}$. Our results support these findings, and it is suggested that AMPK signaling was transiently activated following caffeine treatment but returned to the basal state at $24 \mathrm{~h}$ in the present study.

The AMPK-mediated activation of the ubiquitin-proteasome system is partly dependent on HSP72. In a previous study ${ }^{15}$, we demonstrated that the AMPK-mediated upregulation of MuRF1 mRNA is suppressed in HSP72-knockdown cells. HSP72 is one of the most prominent members of the HSP family, and it is considered to have an important role in controlling skeletal muscle mass ${ }^{34,35}$. HSP72 targets FoxOs, a transcriptional factor of MuRF1 ${ }^{36}$. In the present study, there were no changes in the expression levels of HSP72 protein and mRNA (Figure 3), or in the phosphorylation of FoxO3a $\operatorname{Ser}^{253}$ (Figure 4B). Overall, in contrast to our expectations, our results suggest that caffeine upregulates the ubiquitin-proteasome system via an AMPK-independent mechanism. However, it cannot be excluded the possibility that the transient activation of AMPK might affect the caffeine-mediated responses in the present study. In this regard, further investigations, using a pharmacological blocker or cells with knockdown of AMPK, are necessary to understand the role of AMPK in caffeine-mediated regulation of muscle size.

NF-кB, miR-23a, and myogenin are potent molecules that transcribe MuRF1 and/or atrogin-1/MAFbx. NF- $\kappa \mathrm{B}$ is a transcriptional factor, which is sequestered in the cytoplasm by a family of inhibitory proteins called $\mathrm{I} \kappa \mathrm{B} \alpha^{37}$. The I $\mathrm{I} \mathrm{B}$ kinase complex phosphorylates $\mathrm{I} \kappa \mathrm{B} \alpha$, thereby resulting in its degradation, which leads to the nuclear 


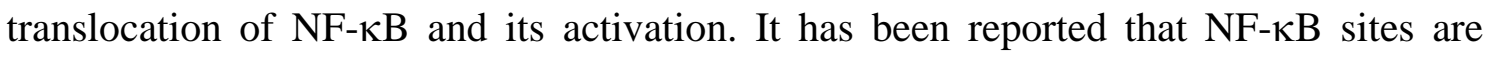
required for the transcriptional activation of MuRF1 during the regulation of muscle mass $^{38}$. However, in the present study, we found that I $\mathrm{B} \alpha$ expression was not affected by caffeine (Figure 4B).

miRs comprise a novel class of small, non-coding endogenous RNAs, which regulate gene expression by directing their target mRNAs for degradation or translational repression ${ }^{39,40}$. Recently, it was shown that miR-23a suppresses post-transcriptional MuRF1 and atrogin-1/MAFbx expressions in vitro and that the forced expression of miR23a in skeletal muscle leads to resistance against atrophy by regulating MuRF1 and atrogin-1/MAFbx genes ${ }^{41}$. In the present study, caffeine did not affect the expression level of miR-23a (Figure 4C).

Myogenin is an essential transcription factor for skeletal muscle development ${ }^{42}$, which binds and activates the promoter regions of the MuRF1 and atrogin-1/MAFbx genes $^{43}$. It has been demonstrated that the upregulation of MuRF1 and atrogin-1/MAFbx in denervated skeletal muscle was impaired in myogenin null mice ${ }^{43}$. In the present study, caffeine upregulated the expression level of myogenin mRNA (Figure 4D), which suggests that caffeine might activate the ubiquitin-proteasome system by increasing myogenin-mediated MuRF1 and atrogin-1/MAFbx transcriptions.

Skeletal muscle mass is regulated by the rate of protein turnover, namely the balance between protein synthesis and degradation ${ }^{44}$. In previous studies ${ }^{7,21}$, we showed that caffeine treatment acutely inhibits the Akt/mTOR/p70S6K signaling in rat skeletal muscle. Correspondingly, in the present study, we confirmed that caffeine decreased phosphorylation of Akt Ser ${ }^{473}$ (Figure 5A) as well as p70S6K Thr ${ }^{389}$ (Figure 5B) in caffeine-treated cells, suggesting that caffeine also affects protein synthesis pathway in 
addition to proteolysis pathway. Taken together, the data obtained the present study suggests that the decreased muscle size (Figure 1) by caffeine might be attributed to the disturbance of protein turnover, including suppression of protein synthesis and stimulating protein degradation.

In terms of exercise, caffeine ingestion elicits high performance with respect to the endurance capacity and muscle strength ${ }^{45}$. However, caffeine might also inhibit protein synthesis and stimulate protein degradation, given that caffeine suppresses the Akt/mTOR/p70S6K signaling pathway as well as promoting the autophagy ${ }^{10}$ and ubiquitin-proteasome pathways. Therefore, caffeine ingestion may possibly affect muscle size: inhibiting muscle hypertrophy or promoting muscle atrophy. Furthermore, it has been shown that caffeine ingestion acutely lowers glucose tolerance in skeletal muscle ${ }^{46}$ by preventing insulin signal transduction ${ }^{7}$. Therefore, caffeine is not necessarily beneficial for muscle functions. To the best of our knowledge, the present study is the first to demonstrate the involvement of caffeine in the regulation of muscle size and the ubiquitin-proteasome system in skeletal muscle. Further research should clarify the effects of caffeine on skeletal muscle size in physiological conditions to obtain a better understanding of the roles of caffeine in skeletal muscle functions. 


\section{CONCLUSIONS}

In this study, we showed that caffeine treatment reduced in myotube size in cultured C2C12 cells and stimulated the ubiquitin-proteasome system. In addition, caffeine treatment did not promote AMPK signaling or its associated molecules, whereas it upregulated myogenin mRNA expression. On the other hand, the protein synthesis pathway was inhibited by caffeine. The results obtained in the present study indicate that caffeine might affect muscle size by stimulating ubiquitin-proteasome system and inhibiting Akt/mTOR/p70S6K signaling, partly via an AMPK-independent mechanism. 


\section{ACKNOWLEDGMENTS}

We thank Liu-Lin Tang, M.D., Ph.D., Toyohashi SOZO University, for technical assistance. This study was supported, in part, by Grants-in-Aid from the Japan Society for the Promotion of Science (JSPS) Fellows (TE, 12J03899; AG, 14J00286), Grants-inAid for Scientific Research from JSPS (TE, 26560371; Y Ohno, 25350641; Y Ohira, 26560373; TY, 26350818; KG, 26560372), the Ministry of Agriculture, Forestry and Fisheries, Integration Research for Agriculture and Interdisciplinary Fields from Biooriented Technology Research Advancement Institution, NARO (TH, 14532022), the Japanese Council for Science, Technology and Innovation, SIP from NARO (TH, 14533567), and research grants from the Uehara Memorial Foundation (KG), the Naito Foundation (KG), and the Graduate School of Health Sciences, Toyohashi SOZO University (KG). 


\section{DISCLOSURES}

The authors state that they have no conflicts of interest to declare. 


\section{REFERENCES}

1. Jensen, TE, Rose, AJ, Hellsten, Y, Wojtaszewski, JF, and Richter, EA, Caffeineinduced $\mathrm{Ca}(2+)$ release increases AMPK-dependent glucose uptake in rodent soleus muscle. Am J Physiol Endocrinol Metab. 2007;293:E286-292.

2. Raney, MA and Turcotte, LP, Evidence for the involvement of CaMKII and AMPK in Ca2+-dependent signaling pathways regulating FA uptake and oxidation in contracting rodent muscle. J Appl Physiol (1985). 2008;104:1366-1373.

3. Egawa, T, Hamada, T, Kameda, N, et al., Caffeine acutely activates 5'adenosine monophosphate-activated protein kinase and increases insulin-independent glucose transport in rat skeletal muscles. Metabolism. 2009;58:1609-1617.

4. Abbott, MJ, Edelman, AM, and Turcotte, LP, CaMKK is an upstream signal of AMP-activated protein kinase in regulation of substrate metabolism in contracting skeletal muscle. Am J Physiol Regul Integr Comp Physiol. 2009;297:R1724-1732.

5. Egawa, T, Hamada, T, Ma, X, et al., Caffeine activates preferentially alpha1isoform of 5'AMP-activated protein kinase in rat skeletal muscle. Acta Physiol (Oxf). 2011;201:227-238.

6. Abbott, MJ, Bogachus, LD, and Turcotte, LP, AMPKalpha2 deficiency uncovers time dependency in the regulation of contraction-induced palmitate and glucose uptake in mouse muscle. J Appl Physiol (1985). 2011;111:125-134.

7. Egawa, T, Tsuda, S, Ma, X, Hamada, T, and Hayashi, T, Caffeine modulates phosphorylation of insulin receptor substrate-1 and impairs insulin signal transduction in rat skeletal muscle. J Appl Physiol (1985). 2011;111:1629-1636.

8. Takimoto, M, Takeyama, M, and Hamada, T, Possible involvement of AMPK in acute exercise-induced expression of monocarboxylate transporters MCT1 and MCT4 
mRNA in fast-twitch skeletal muscle. Metabolism. 2013;62:1633-1640.

9. Ding, S, Riddoch-Contreras, J, Abramov, AY, Qi, Z, and Duchen, MR, Mild stress of caffeine increased mtDNA content in skeletal muscle cells: the interplay between Ca2+ transients and nitric oxide. J Muscle Res Cell Motil. 2012;33:327-337.

10. Mathew, TS, Ferris, RK, Downs, RM, Kinsey, ST, and Baumgarner, BL, Caffeine promotes autophagy in skeletal muscle cells by increasing the calciumdependent activation of AMP-activated protein kinase. Biochem Biophys Res Commun. 2014;453:411-418.

11. Thomson, DM and Gordon, SE, Diminished overload-induced hypertrophy in aged fast-twitch skeletal muscle is associated with AMPK hyperphosphorylation. J Appl Physiol (1985). 2005;98:557-564.

12. Gordon, SE, Lake, JA, Westerkamp, CM, and Thomson, DM, Does AMPactivated protein kinase negatively mediate aged fast-twitch skeletal muscle mass? Exerc Sport Sci Rev. 2008;36:179-186.

13. Mounier, R, Lantier, L, Leclerc, J, et al., Important role for AMPKalpha1 in limiting skeletal muscle cell hypertrophy. Faseb J. 2009;23:2264-2273.

14. Miyazaki, M and Esser, KA, Cellular mechanisms regulating protein synthesis and skeletal muscle hypertrophy in animals. J Appl Physiol (1985). 2009;106:1367-1373. 15. Egawa, T, Ohno, Y, Goto, A, et al., AICAR-induced activation of AMPK negatively regulates myotube hypertrophy through the HSP72-mediated pathway in C2C12 skeletal muscle cells. Am J Physiol Endocrinol Metab. 2014;306:E344-354.

16. Bodine, SC, Latres, E, Baumhueter, S, et al., Identification of ubiquitin ligases required for skeletal muscle atrophy. Science. 2001;294:1704-1708.

17. Gomes, MD, Lecker, SH, Jagoe, RT, Navon, A, and Goldberg, AL, Atrogin-1, a 
muscle-specific F-box protein highly expressed during muscle atrophy. Proc Natl Acad Sci U S A. 2001;98:14440-14445.

18. Krawiec, BJ, Nystrom, GJ, Frost, RA, Jefferson, LS, and Lang, CH, AMPactivated protein kinase agonists increase mRNA content of the muscle-specific ubiquitin ligases MAFbx and MuRF1 in C2C12 cells. Am J Physiol Endocrinol Metab. 2007;292:E1555-1567.

19. Nakashima, K and Yakabe, Y, AMPK activation stimulates myofibrillar protein degradation and expression of atrophy-related ubiquitin ligases by increasing FOXO transcription factors in C2C12 myotubes. Biosci Biotechnol Biochem. 2007;71:16501656.

20. Egawa, T, Goto, A, Ohno, Y, et al., Involvement of AMPK in regulating slowtwitch muscle atrophy during hindlimb unloading in mice. Am J Physiol Endocrinol Metab. 2015;309:E651-662.

21. Tsuda, S, Egawa, T, Kitani, K, et al., Caffeine and contraction synergistically stimulate 5'-AMP-activated protein kinase and insulin-independent glucose transport in rat skeletal muscle. Physiol Rep. 2015;3:e12592.

22. Williamson, DL, Butler, DC, and Alway, SE, AMPK inhibits myoblast differentiation through a PGC-1alpha-dependent mechanism. Am J Physiol Endocrinol Metab. 2009;297:E304-314.

23. Ohno, Y, Yamada, S, Sugiura, T, et al., Possible role of NF-kB signals in heat stress-associated increase in protein content of cultured C2C12 cells. Cells Tissues Organs. 2011;194:363-370.

24. Yasuhara, K, Ohno, Y, Kojima, A, et al., Absence of heat shock transcription factor 1 retards the regrowth of atrophied soleus muscle in mice. J Appl Physiol (1985). 
2011;111:1142-1149.

25. Stein, SC, Woods, A, Jones, NA, Davison, MD, and Carling, D, The regulation of AMP-activated protein kinase by phosphorylation. Biochem J. 2000;345 Pt 3:437-443. 26. Brunet, A, Bonni, A, Zigmond, MJ, et al., Akt promotes cell survival by phosphorylating and inhibiting a Forkhead transcription factor. Cell. 1999;96:857-868.

27. Alessi, DR, Andjelkovic, M, Caudwell, B, et al., Mechanism of activation of protein kinase B by insulin and IGF-1. EMBO J. 1996;15:6541-6551.

28. Pearson, RB, Dennis, PB, Han, JW, et al., The principal target of rapamycininduced p70s6k inactivation is a novel phosphorylation site within a conserved hydrophobic domain. EMBO J. 1995;14:5279-5287.

29. Masiero, E, Agatea, L, Mammucari, C, et al., Autophagy is required to maintain muscle mass. Cell Metab. 2009;10:507-515.

30. Komander, D, The emerging complexity of protein ubiquitination. Biochem Soc Trans. 2009;37:937-953.

31. Kim, HT, Kim, KP, Lledias, F, et al., Certain pairs of ubiquitin-conjugating enzymes (E2s) and ubiquitin-protein ligases (E3s) synthesize nondegradable forked ubiquitin chains containing all possible isopeptide linkages. J Biol Chem. 2007;282:17375-17386.

32. Mei, Z, Zhang, D, Hu, B, et al., FBXO32 Targets c-Myc for Proteasomal Degradation and Inhibits c-Myc Activity. J Biol Chem. 2015;290:16202-16214.

33. Park, S, Scheffler, TL, and Gerrard, DE, Chronic high cytosolic calcium decreases AICAR-induced AMPK activity via calcium/calmodulin activated protein kinase II signaling cascade. Cell Calcium. 2011;50:73-83.

34. Gehrig, SM, van der Poel, C, Sayer, TA, et al., Hsp72 preserves muscle function 
and slows progression of severe muscular dystrophy. Nature. 2012;484:394-398.

35. Senf, SM, Skeletal muscle heat shock protein 70: diverse functions and therapeutic potential for wasting disorders. Front Physiol. 2013;4:330.

36. Waddell, DS, Baehr, LM, van den Brandt, J, et al., The glucocorticoid receptor and FOXO1 synergistically activate the skeletal muscle atrophy-associated MuRF1 gene. Am J Physiol Endocrinol Metab. 2008;295:E785-797.

37. Mourkioti, F and Rosenthal, N, NF-kappaB signaling in skeletal muscle: prospects for intervention in muscle diseases. J Mol Med (Berl). 2008;86:747-759.

38. Wu, CL, Cornwell, EW, Jackman, RW, and Kandarian, SC, NF-kappaB but not FoxO sites in the MuRF1 promoter are required for transcriptional activation in disuse muscle atrophy. Am J Physiol Cell Physiol. 2014;306:C762-767.

39. Bartel, DP, MicroRNAs: genomics, biogenesis, mechanism, and function. Cell. 2004;116:281-297.

40. Guller, I and Russell, AP, MicroRNAs in skeletal muscle: their role and regulation in development, disease and function. J Physiol. 2010;588:4075-4087.

41. Wada, S, Kato, Y, Okutsu, M, et al., Translational suppression of atrophic regulators by microRNA-23a integrates resistance to skeletal muscle atrophy. J Biol Chem. 2011;286:38456-38465.

42. Hasty, P, Bradley, A, Morris, JH, et al., Muscle deficiency and neonatal death in mice with a targeted mutation in the myogenin gene. Nature. 1993;364:501-506.

43. Moresi, V, Williams, AH, Meadows, E, et al., Myogenin and class II HDACs control neurogenic muscle atrophy by inducing E3 ubiquitin ligases. Cell. 2010;143:3545.

44. Goldspink, DF, Garlick, PJ, and McNurlan, MA, Protein turnover measured in 
vivo and in vitro in muscles undergoing compensatory growth and subsequent denervation atrophy. Biochem J. 1983;210:89-98.

45. Shearer, J and Graham, TE, Performance effects and metabolic consequences of caffeine and caffeinated energy drink consumption on glucose disposal. Nutr Rev. 2014;72 Suppl 1:121-136.

46. Thong, FS, Derave, W, Kiens, B, et al., Caffeine-induced impairment of insulin action but not insulin signaling in human skeletal muscle is reduced by exercise. Diabetes. 2002;51:583-590. 


\section{FIGURE LEGENDS}

Figure 1

Effects of caffeine on the: (A) protein content, (B) myotube diameter, and (C) C2C12 myotube diameter distribution. Myotubes were incubated in differentiation medium without or with caffeine (A: indicated concentration; B and C: $1 \mathrm{mM}$ ) for $24 \mathrm{~h}$. Representative images of myotubes are also shown. Scale bars indicate $100 \mu \mathrm{m}$. Values represent the mean \pm SEM. $\mathrm{n}=6$ per group. ${ }^{*}: P<0.05$ vs. no treatment.

Figure 2

Effects of caffeine on the expression levels of: (A) MuRF1 mRNA, (B) atrogin1/MAFbx mRNA and (C) K48-linked polyubiquitin (K48-Ub) in C2C12 myotubes. Myotubes were incubated in differentiation medium without or with caffeine (1 mM) for 24 h. Representative immunoblots are also shown. Values represent the mean \pm SEM. $n$ = 3-6 per group. $*: P<0.05$ vs. no treatment.

Figure 3

Effects of caffeine, AICAR, or metformin on the expression levels of: (A) phosphorylated AMPK Thr ${ }^{172}$ (p-AMPK)/AMPK, (B) HSP72, and (C) HSP72 mRNA in C2C12 myotubes. Myotubes were incubated in differentiation medium without or with caffeine (1 mM), AICAR (0.5 mM), and metformin (2 mM) for 24 h. Representative immunoblots are also shown. Values represent the mean \pm SEM. $\mathrm{n}=6$ per group. ${ }^{*}: \mathrm{P}<$ 0.05 vs. no treatment.

Figure 4 


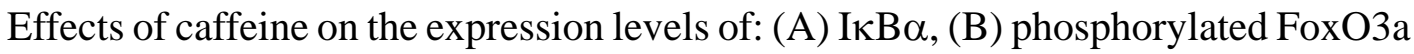
$\operatorname{Ser}^{253}$ (p-FoxO3a)/FoxO3a, (C) miR-23a, and (D) myogenin mRNA in C2C12 myotubes. Myotubes were incubated in differentiation medium without or with caffeine (1 mM) for

24 h. Representative immunoblots are also shown. Values represent the mean \pm SEM. $n$ $=6$ per group. ${ }^{*}: P<0.05$ vs. no treatment.

Figure 5

Effects of caffeine on the expression levels of: (A) phosphorylated Akt $\operatorname{Ser}^{473}$ (pAkt)/Akt and (B) phosphorylated p70S6K $\mathrm{Thr}^{389}$ (p-p70S6K)/p70S6K in C2C12 myotubes. Myotubes were incubated in differentiation medium without or with caffeine (1 mM) for 24 h. Representative immunoblots are also shown. Values represent the mean \pm SEM. $\mathrm{n}=6$ per group. ${ }^{*}: P<0.05$ vs. no treatment. 
A

B

protein content
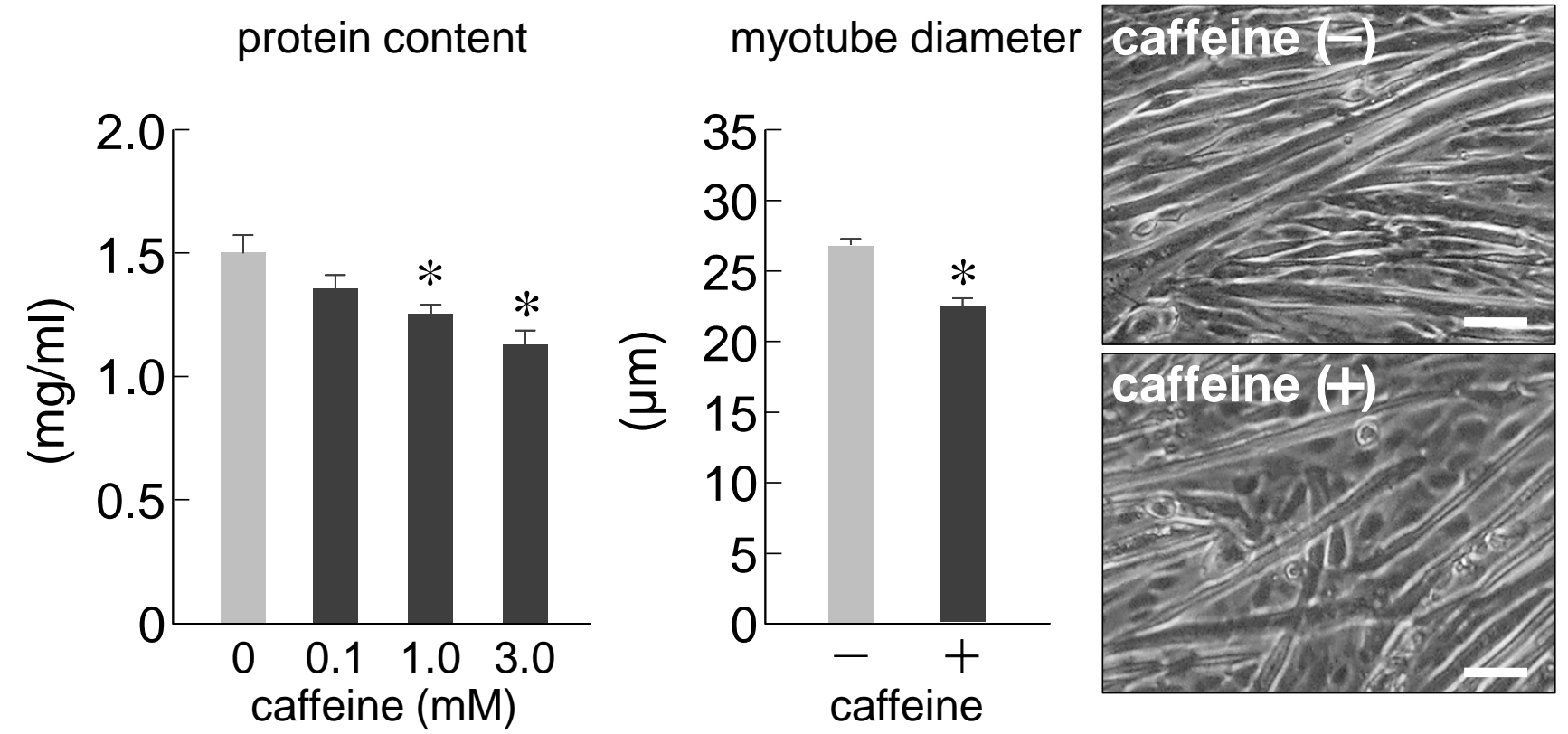

C

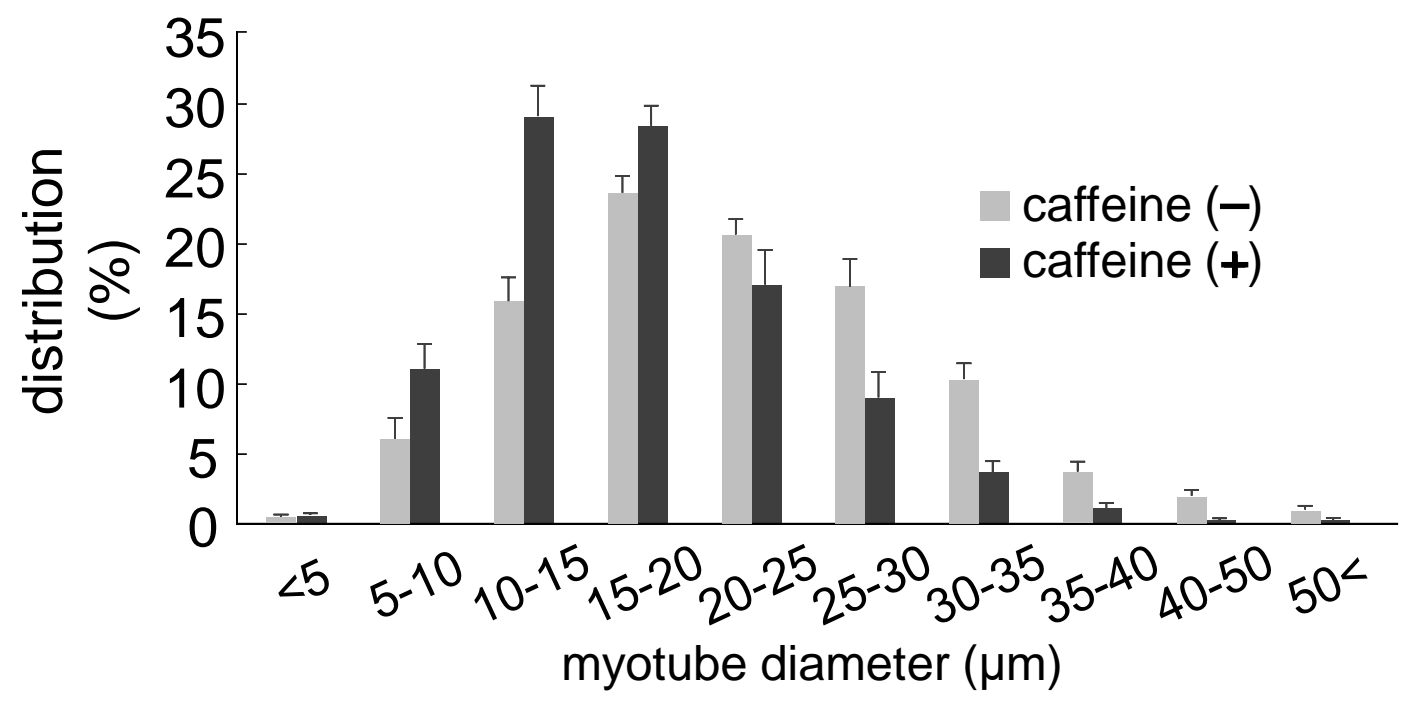

Figure 1 
A

B

C

MuRF1 mRNA

atrogin-1/MAFbx K48-Ub

$\beta$-actin
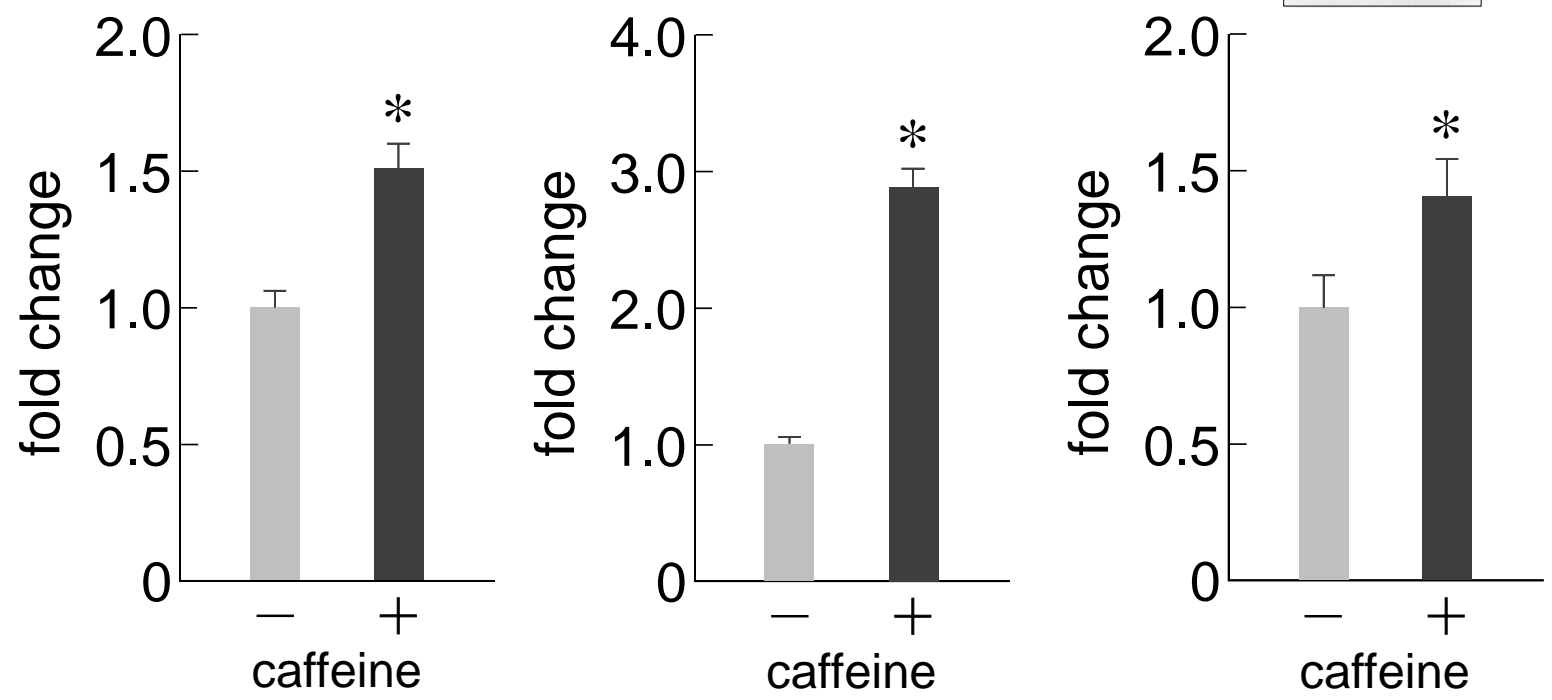

Figure 2 


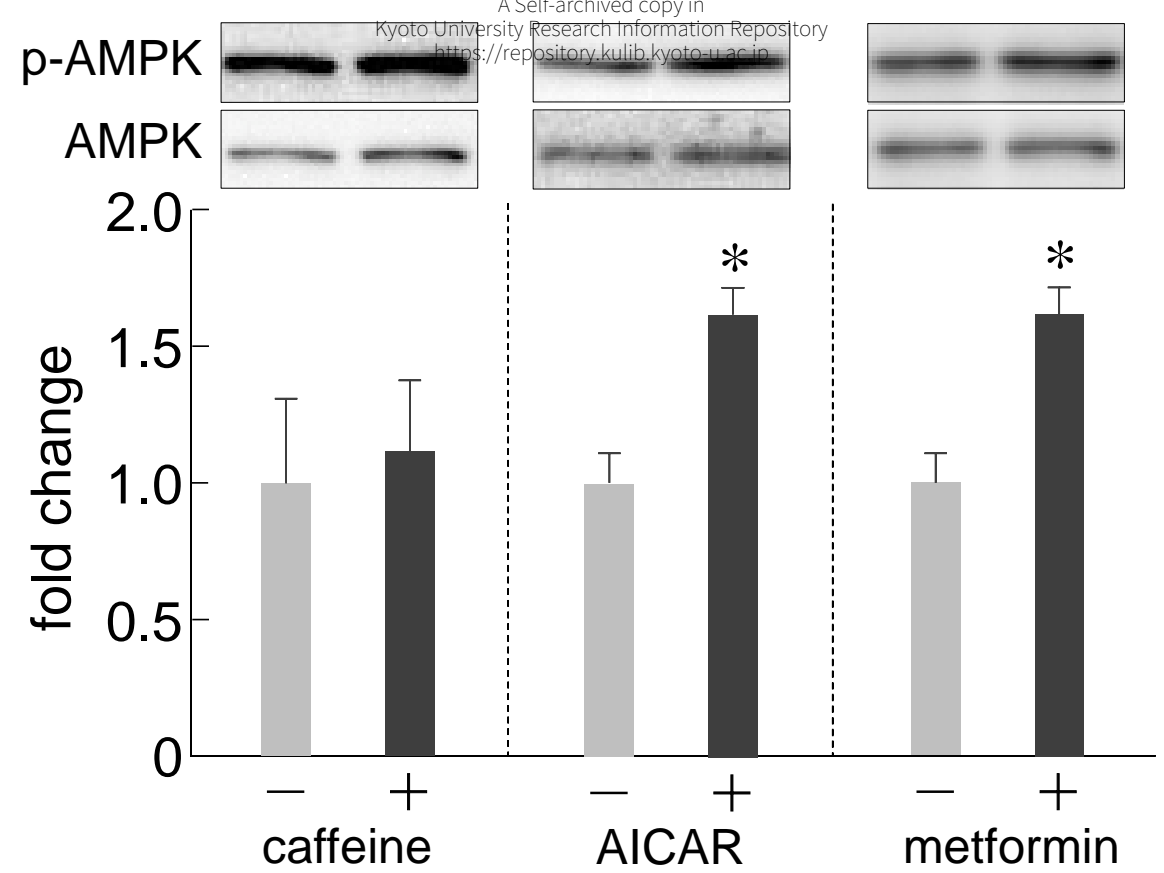

B

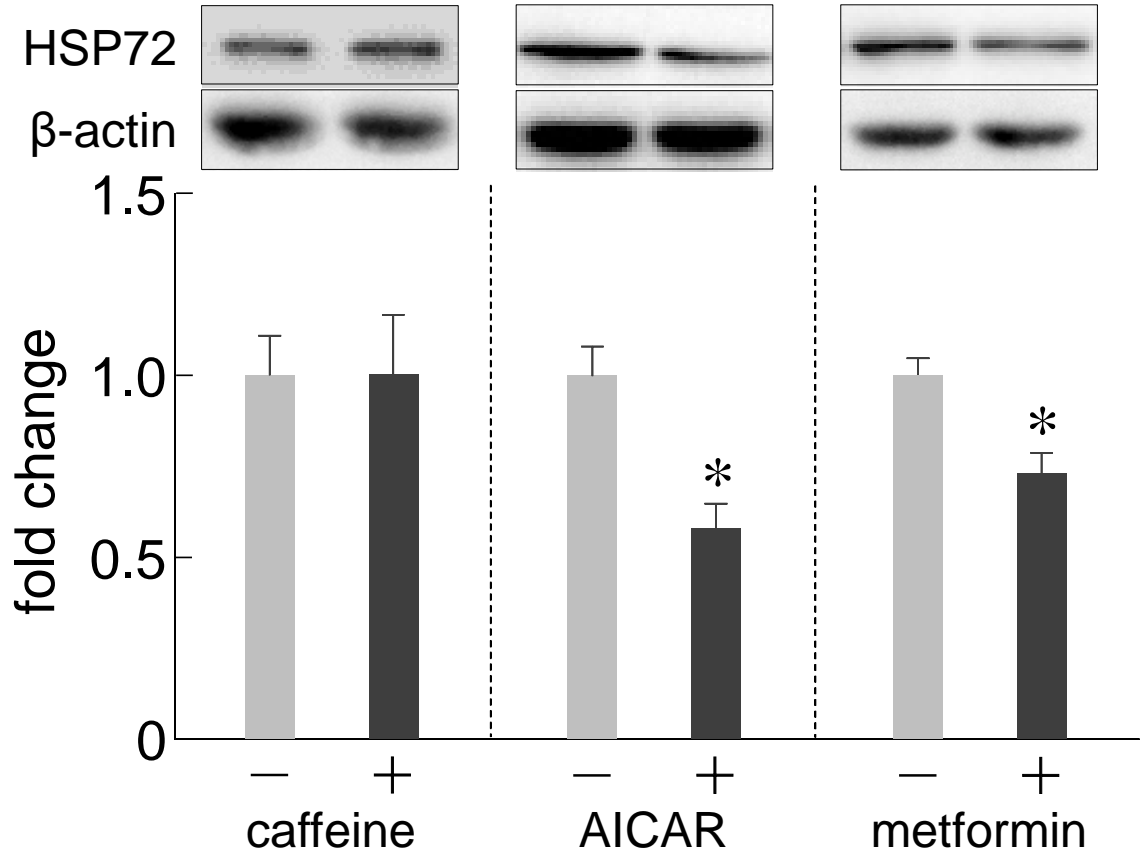

C HSP72 mRNA

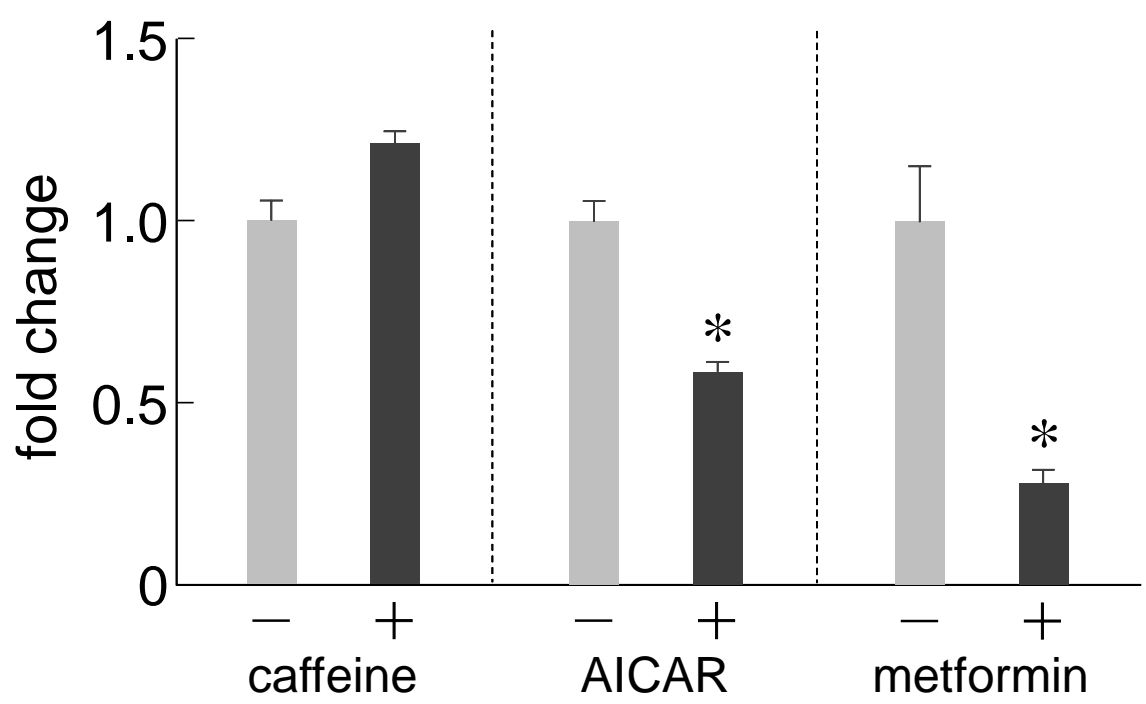

Figure 3 
A

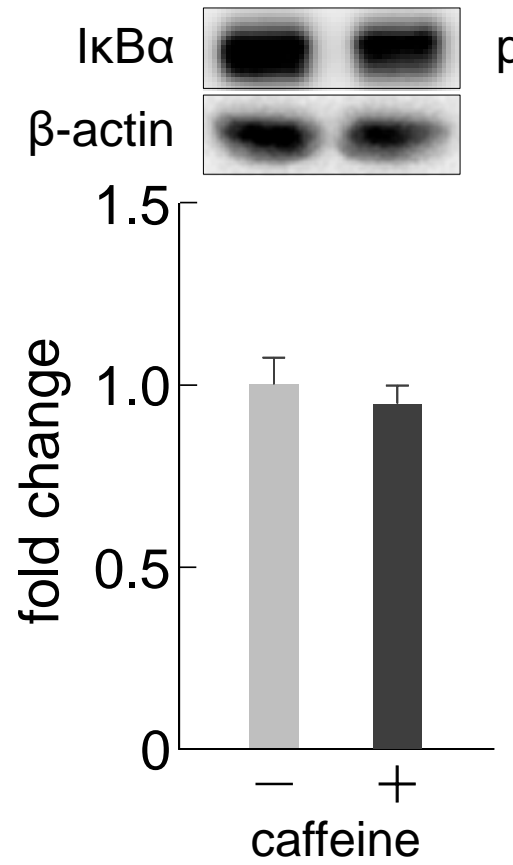

C

$\operatorname{miR}-23 a$

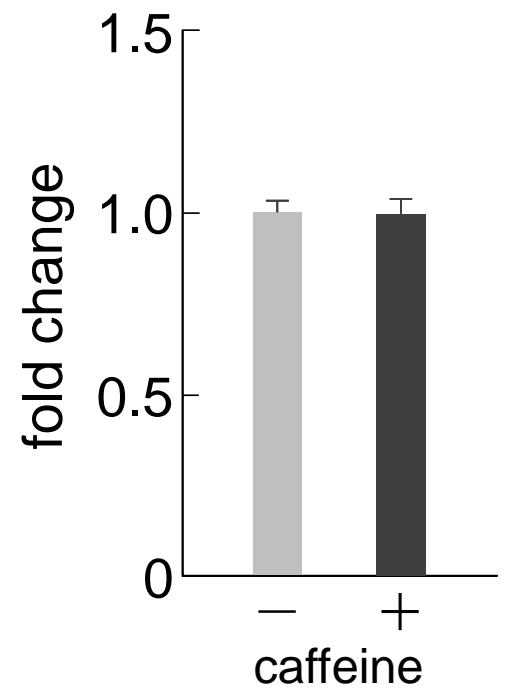

B

p-FoxO3a

FoxO3a

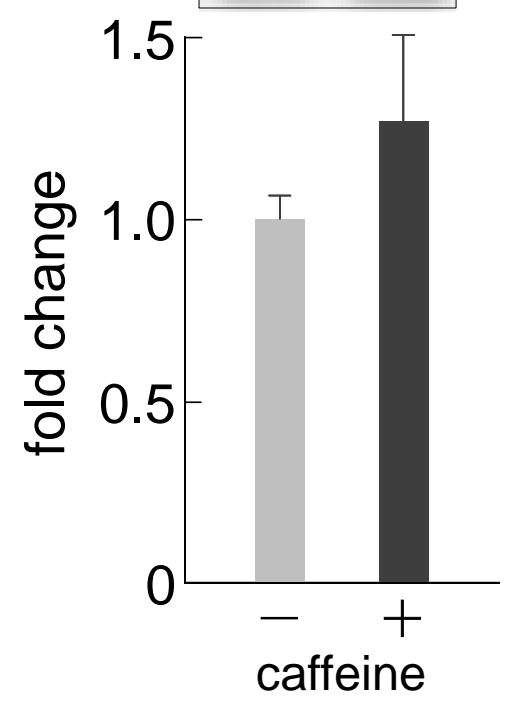

D

myogenin mRNA

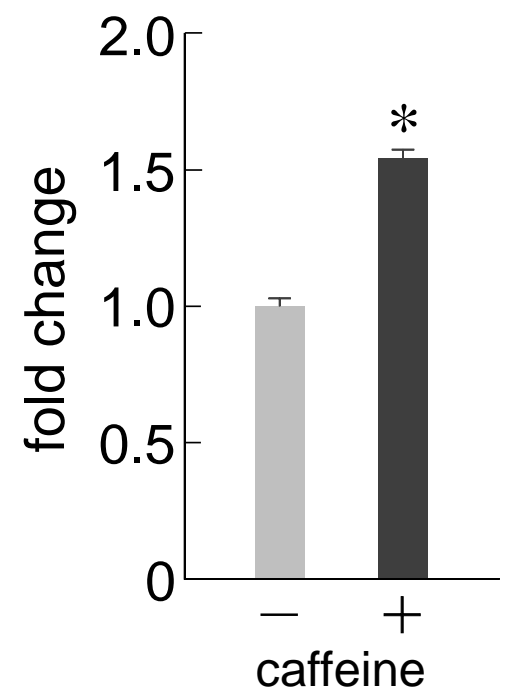

Figure 4 
A

B

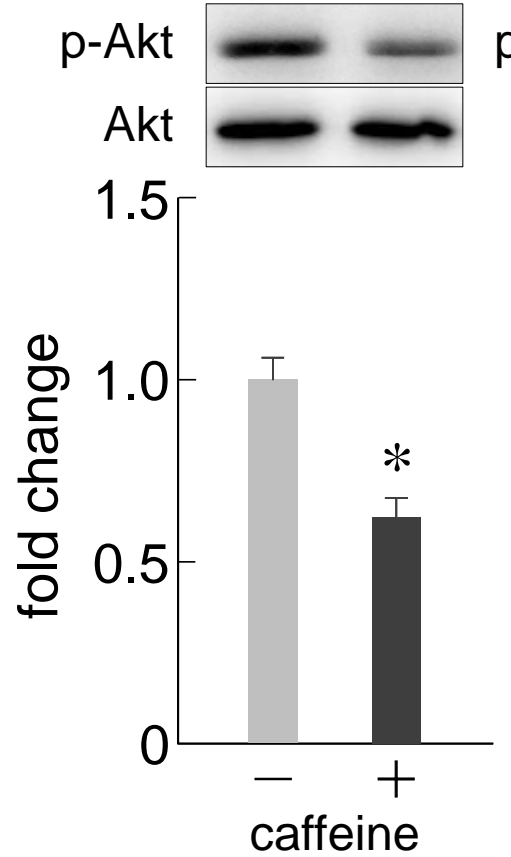

$p-p 70 S 6 K$
$p 70 S 6 K$

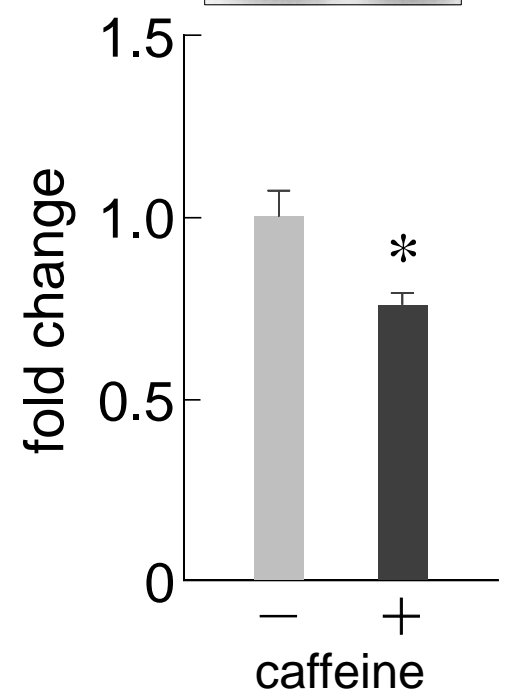

Figure 5 